\title{
Unilateral coronal synostosis: can we trust the sagittal suture as a landmark for the underlying superior sagittal sinus?
}

\author{
Tatiana Protzenko Cervante, MD, ${ }^{1}$ Eric Arnaud, MD, ${ }^{1}$ Francis Brunelle, MD, $\mathrm{PhD},{ }^{2}$ and \\ Federico Di Rocco, MD1
}

Departments of ${ }^{1}$ Pediatric Neurosurgery and ${ }^{2}$ Neuroradiology, Necker Enfants Malades Hospital, APHP, Paris, France

\begin{abstract}
OBJECTIVE The sagittal suture is usually considered an external anatomical landmark, indicating the location of the superior sagittal sinus (SSS) for surgical approaches. Children with unilateral coronal synostosis (UCS) often present with an important deviation of the sagittal suture. Because these patients usually undergo frontal reconstruction or even endoscope-assisted minimally invasive procedures, it is imperative to know the location of the SSS. The aim of this investigation was to study the anatomical relationship between the SSS and the sagittal suture in children with anterior plagiocephaly.
\end{abstract}

METHODS The authors retrospectively studied the relationship between the sagittal sinus and the sagittal suture at 5 points: nasion, midpoint nasion-bregma, bregma, midpoint bregma-lambda, and lambda. The study analyzed CT scans of 50 children with UCS admitted to the craniofacial unit of Necker Enfants Malades Hospital between March 2006 and March 2013 and compared them with 50 control children with no evidence of craniosynostosis, bone disease, or genetic syndromes. The authors also analyzed the presence of extracerebral fluid collection and ventricular asymmetry in children with UCS.

RESULTS Fifty-six percent of patients had anterior right UCS and $44 \%$ had left-sided UCS. Type I UCS was seen in 1 patient, Type IIA in 20 patients, Type IIB in 20 patients, and Type III in 9 patients. The authors found that the nasion is usually deviated to the ipsilateral side of the synostosis, the bregma contralaterally, and the lambda ipsilaterally. The gap distances between the reference point and the SSS were 0-7.3 mm (mean $1.4 \mathrm{~mm}$ ) at the nasion; 0-16.7 mm (mean 3.8 $\mathrm{mm}$ ) at the midpoint nasion-bregma; 0-12 mm (mean $5.8 \mathrm{~mm}$ ) at the bregma; 0-9.5 mm (mean $3 \mathrm{~mm}$ ) at the midpoint bregma-lambda; and 0-11.6 $\mathrm{mm}$ (mean $5.5 \mathrm{~mm}$ ) at the lambda. Conversely, a discrepancy of more than $1 \mathrm{~mm}$ between the SSS and the position of the suture was found only in 7 control cases (14\%).

Of patients with UCS, $38 \%$ presented with an extracerebral fluid collection contralateral to the fused coronal suture. Fifty-two percent had a ventricular asymmetry, which was characterized by reduced ventricular volume ipsilateral to the synostosis in all but 1 patient.

CONCLUSIONS In this study, the SSS was usually deviated contralaterally to the closed coronal suture. It tended to be in the midline of the cranial vault and could be projected virtually along an imaginary line passing through the midline of the cranial base. The authors recommend a distance of $37 \mathrm{~mm}$ from the sagittal suture as a safety margin during surgery.

http://thejns.org/doi/abs/10.3171/2015.8.PEDS15117

KEY WORDS plagiocephaly; superior sagittal sinus; cranial suture; unilateral coronal synostosis; venous sinus; coronal suture; craniofacial

$\mathrm{C}$ RANIOSYNOSTOSIS is defined as the premature closure of cranial vault sutures. ${ }^{4}$ It is a rare congenital defect affecting less than 1 in 2000-2500 live births worldwide. ${ }^{18}$ Craniosynostosis usually occurs as an isolated condition, representing approximately $70 \%$ of all craniosynostosis, but can also manifest in association with a syndrome. ${ }^{9}$
Plagiocephaly due to unilateral coronal synostosis (UCS) is currently considered the third most common type of simple craniosynostosis, following scaphocephaly and trigonocephaly, with an incidence of 13\%-16\% among patients with craniosynostosis. . $^{1,2,3}$

The treatment of this condition usually requires an open frontal-orbital advancement based on a bifrontal craniot-

ABBREVIATIONS SSS = superior sagittal sinus; UCS = unilateral coronal synostosis.

SUBMITTED February 22, 2015. ACCEPTED August 27, 2015.

INCLUDE WHEN CITING Published online January 8, 2016; DOI: 10.3171/2015.8.PEDS15117. 
omy. The parietal bone may sometimes be used as an alternative if it is better shaped to the bifrontal contour. ${ }^{10,11,13}$ This surgical method requires elevation of the skull bones at the level of the forehead and, in some cases, more posteriorly, in close relationship with the superior sagittal sinus (SSS). The SSS courses in the midline beginning just behind the frontal bone and grows larger as it continues posteriorly along the shallow groove on the inner table of the cranium. ${ }^{14}$ Today, some authors ${ }^{6}$ have changed the approach and have begun to treat patients at the earliest age possible, using endoscope-assisted minimally invasive procedures. However, even in this minimally invasive way, there is proximity to the SSS. Disruption of the SSS can lead to serious complications, such as cerebral vein infarction, uncontrolled bleeding, intracranial hypertension, and even death. Due to this surgical challenge, it is essential that the neurosurgeon estimates the correct location of the SSS.

Generally, the SSS is considered to be under the sagittal suture, which is considered an external landmark of SSS between the bregma and the lambda. ${ }^{14}$ However, some authors have questioned this anatomical relationship, ${ }^{6,16,19}$ and further studies are needed. The aim of the present study is 3-fold: 1) to study the position of the SSS in relation to the sagittal suture in 50 patients with UCS; 2) to compare this anatomical relationship between patients with UCS and 50 control children without craniosynostosis; and 3) to study the presence of extracerebral fluid collection and ventricular asymmetry in patients with UCS to see if there is a correlation with the degree of SSS displacement.

\section{Methods}

We retrospectively analyzed $50 \mathrm{CT}$ scans of children with UCS who were admitted to the craniofacial unit of Necker Enfants Malades Hospital between March 2006 and March 2013. The relationship between the sagittal sinus and the sagittal suture were studied at 5 points: 1 ) nasion-studied at axial plane of CT scan; 2) midpoint nasion-bregma-studied at axial plane of CT scan; 3) bregma-studied at coronal plane of CT scan; 4) midpoint bregma-lambda-studied at coronal plane of CT scan; and 5) lambda-studied at axial plane of CT scan.

Before any measurement, all CT scans were reconstructed in multiplanar projections to align the images according to the auriculo-orbital plane. From this orientation, we performed the cuts of the skull base and calvaria. The midline was considered the imaginary line that passes through the middle of the sella, represented in Fig. 1. All patients with UCS were classified into Types I, IIA, IIB, and III, according to the proposed classification by Di Rocco et al. ${ }^{3}$ Type I is characterized by unilateral flattening of the frontal bone and elevation of the superior orbital ridge without deviation of the nasal pyramid. Type II refers to the deviation of the nasal pyramid and homolateral anterior displacement of the petrous bone (Types IIA and IIB, according to the severity of petrous bone displacement). Type III is characterized by severe deviation of the sphenobasilar bone. Distances were measured from the edge of the sagittal suture to the far edge of the SSS, so that we could define a safety margin during surgery

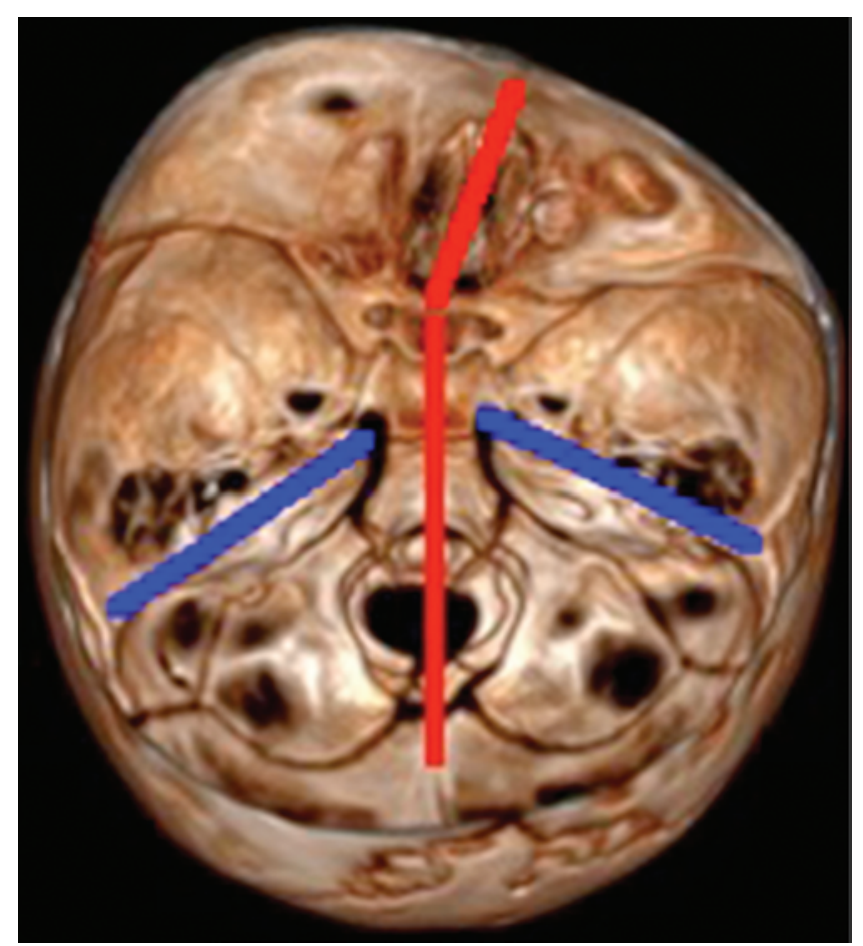

FIG. 1. Right-sided coronal synostosis Type IIB. The imaginary midline passes through the midline of the sella to the opisthion. Figure is available in color online only.

(Fig. 2). After measurement of the SSS regarding the 5 reference points mentioned above, the sinus was designed over the 3D reconstruction to represent its deviation from the sagittal suture. In the UCS group, we also determined whether extracerebral fluid collection was present contralateral to the synostosis (Fig. 3) and if there was a ventricular asymmetry.

The group of patients with UCS was then compared with 50 control children with nonaccidental brain injury and no evidence of craniosynostosis, bone disease, or genetic syndromes. Patients with brain lesions, hematomas, and/or midline shift were excluded from the control group. To compare the measures of the UCS group with the control group, we performed an unpaired t-test. For the variables sex and age, the groups were compared using Fisher's exact test. Consent to perform the studies was obtained from all patients' families.

\section{Results}

\section{Study Population}

Of the 50 patients with UCS, $56 \%$ (28) had fusion of the right coronal suture and 44\% (22) had left-sided fusion. Type I UCS was seen in only 1 patient, Type IIA in 20 patients, Type IIB in 20 patients, and Type III in 9 patients.

The ages of patients with UCS ranged from 0.13 to 21 months at the time of CT scan (mean age 8.2 months). The mean age of children in the control group was 5.5 months, ranging from 1 to 12 months.

\section{Five Landmarks-UCS Group}

We noticed that the nasion and the lambda were usually 


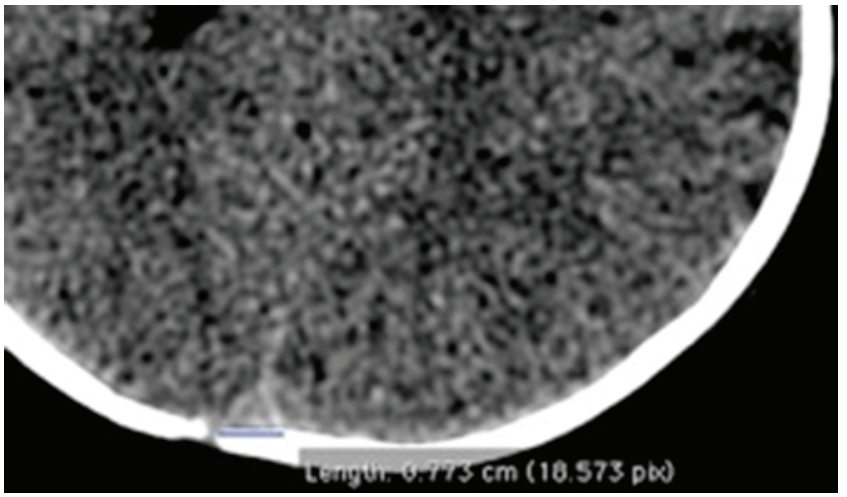

FIG. 2. Example of measurement at the lambda in axial CT scan. Distance is from the edge of the suture to the far edge of the SSS. Figure is available in color online only.

deviated to the ipsilateral side of the synostosis, whereas the bregma was deviated contralaterally. The sagittal suture tends to begin on the opposite side of the UCS at the level of bregma and tends to reapproach the same side of the synostosis when it reaches the lambda, making a diagonal path (Fig. 4). Comparisons of distances found between the midline sutures (metopic in the front and then sagittal) and the SSS in patients with UCS and control groups are reported in Table 1.

At nasion, the first reference point, the distances ranged from 0 to $7.3 \mathrm{~mm}$ (mean $1.4 \mathrm{~mm}$ ). In 31 patients $(62 \%)$, the sinus was just under the metopic suture at this point, but when dislocated (in 38\% of patients), the sinus tended to be located at the opposite side of the synostosis, with only 2 exceptions. These findings demonstrate that in a right-sided plagiocephaly, the sinus is displaced to the left of the suture.

At the midpoint nasion-bregma, the distances ranged from 0 to $16.7 \mathrm{~mm}$ (mean $3.8 \mathrm{~mm}$ ). Sixty-six percent of patients (33) presented with sinus displacement. In $94 \%$ of these patients, the sinus was displaced to the opposite side of the synostosis, with only 2 exceptions. For example, in a left coronal synostosis, the nasion-bregma tends to be near the left side; thus, the sinus runs to the right side of the sagittal suture at a distance of $7.4 \mathrm{~mm}$. The remaining $34 \%$ of patients (17) did not present with sinus displacement (i.e., the sinus was located just below the suture).

Regarding the bregma, 40 patients $(80 \%)$ had a sinus displacement. Among them, $95 \%$ presented with the sinus dislocated to the same side of the synostosis, at a distance ranging from 0 to $12 \mathrm{~mm}$ (mean $5.8 \mathrm{~mm}$ ). Only 1 patient presented with the sinus displaced ipsilaterally to the UCS.

At the level of the midpoint bregma-lambda, the displacement distances ranged from 0 to $9.5 \mathrm{~mm}$ (mean 3.0 $\mathrm{mm}$ ). Twenty-seven patients presented with the sinus dislocated to the contralateral side of the UCS. Only 3 patients presented with the sinus displaced to the same side of the synostosis. The remaining 20 patients had no displacement.

In relation to the lambda, $78 \%$ of patients presented with a sinus displacement, all to the opposite side of the synostosis. These distances ranged from 0 to $11.6 \mathrm{~mm}$ (mean $5.5 \mathrm{~mm}$ ).
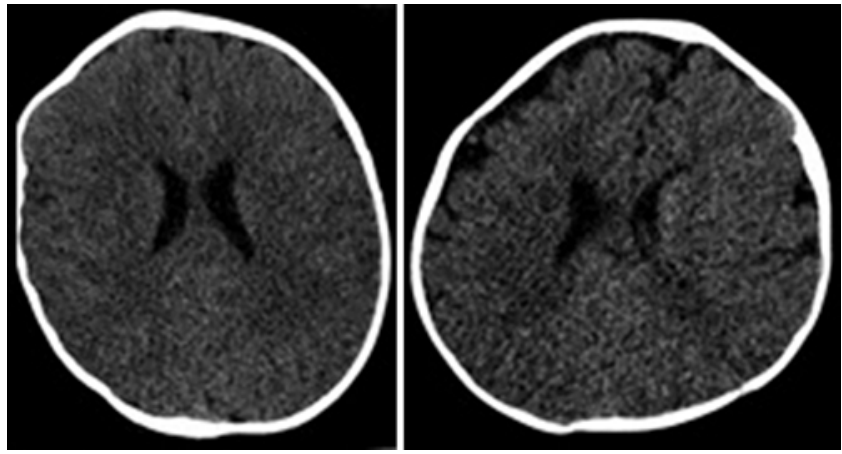

FIG. 3. Classification of the extracerebral space. Right UCS without extracerebral fluid collection (normal) (left panel). Left UCS with a small volume of extracerebral fluid collection contralateral to the synostosis (right panel).

Thus, as the metopic and sagittal sutures course in a diagonal fashion, with the nasion and lambda dislocated ipsilaterally to the synostosis and the bregma contralaterally, the SSS has a tendency to try to reach the virtual midline, crossing these sutures many times. Figure 5 represents the frequency of sinus displacement at the 5 landmarks.

\section{Control Group}

As expected, the SSS was just below the suture at the nasion and at the midpoint nasion-bregma. Interestingly, at the level of the bregma, 2 patients (4\%) presented with a deviation of the sinus of up to $4.7 \mathrm{~mm}$ (mean $0.2 \mathrm{~mm}$ ). In these 2 cases, the sinus was displaced to the right of the sagittal suture. Regarding the midpoint bregma-lambda, only 3 patients presented with a displaced SSS, all of them to the right side, at a distance up to $7.1 \mathrm{~mm}$ (mean $0.3 \mathrm{~mm}$ ). Also, 3 patients presented with sinus displacement relative to the lambda, up to $7 \mathrm{~mm}$ (mean $0.3 \mathrm{~mm}$ ) and always to the right side.

The maximum gap between the sinus and the suture was thus $7.1 \mathrm{~mm}$. Overall, a discrepancy of more than 1 $\mathrm{mm}$ between the SSS and the position of the suture was found in 7 control cases (14\%). None of the patients presented with an extracerebral fluid collection or ventricular asymmetry.

\section{Patients With UCS Versus Control Group}

Regarding sex, Fisher's exact test was performed and the 2 -tailed $\mathrm{p}$ value was 0.1612 , showing that the association between groups and outcomes is not considered statistically significant. For age, the 2-tailed $\mathrm{p}$ value was less than 0.0001 . The UCS group (mean age 8.2 months, range 0.13-21 months) was older than the control group (mean age 5.5 months, range $1-12$ months). However, this difference did not seem to affect our results.

To compare the UCS group with the control group, we performed an unpaired t-test. Table 1 shows the differences between the means of the 2 groups. The differences among all landmark points were considered statistically significant. The most important gaps were found at the levels of the lambda and bregma in patients with UCS, whereas it was at the midpoint bregma-lambda in control subjects (Fig. 6). 


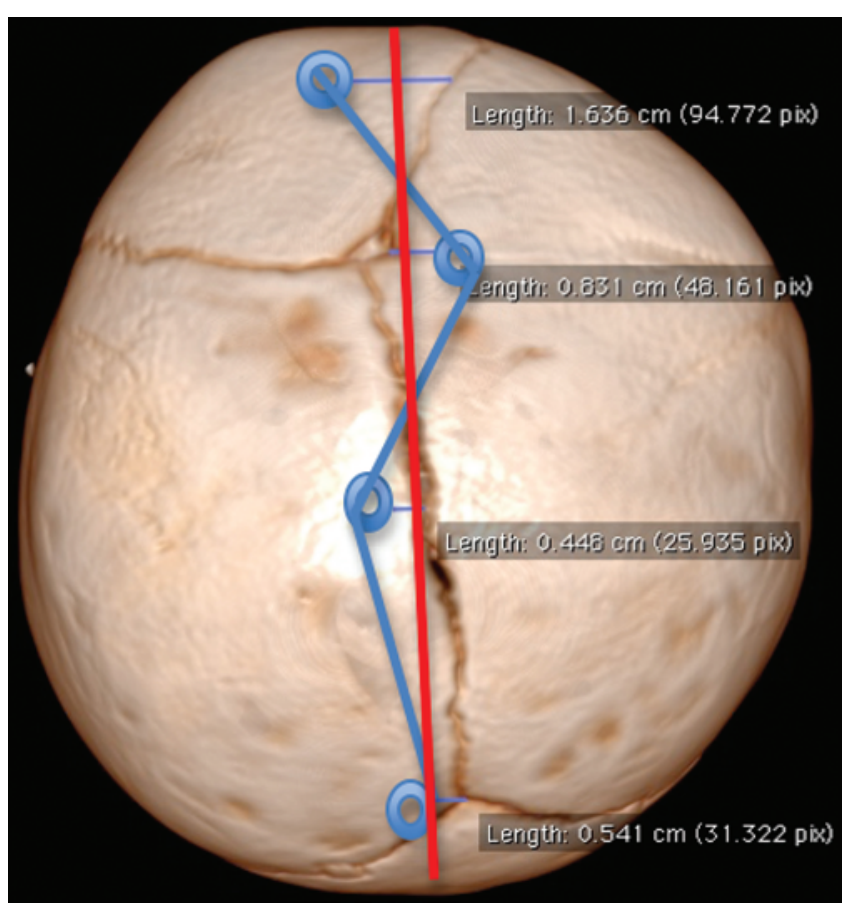

FIG. 4. Right-sided coronal synostosis. Distances from the metopic and sagittal sutures at nasion-bregma, bregma, bregma-lambda, and lambda. The SSS (blue line) makes a path contralateral to the sutures, crossing them 2 times and the imaginary midline (red line) 3 times. Figure is available in color online only.

\section{Extracerebral Space and Ventricular Asymmetry in Patients With UCS}

Extracerebral Space

Nineteen patients $(38 \%)$ presented with extracerebral fluid collection, which was located to the contralateral side of the synostosis in all cases. At some point, all of these patients presented with an SSS deviation larger than $7.1 \mathrm{~mm}$ (i.e., the maximum gap measured in the control group). However, we also found large distances in patients without extracerebral fluid collection. No causal relationship between these features could be found (Fig. 3).

\section{Ventricular Symmetry}

Ventricular symmetry was also evaluated. We found that $50 \%$ of patients with UCS (25) presented with a ventricular asymmetry; $96 \%$ of these patients presented with small ventricles on the same side of the synostosis. Of them, 17 presented with an SSS deviated more than 7.1 $\mathrm{mm}$. However, we found patients with more severe deviation without ventricular asymmetry; a causal relationship

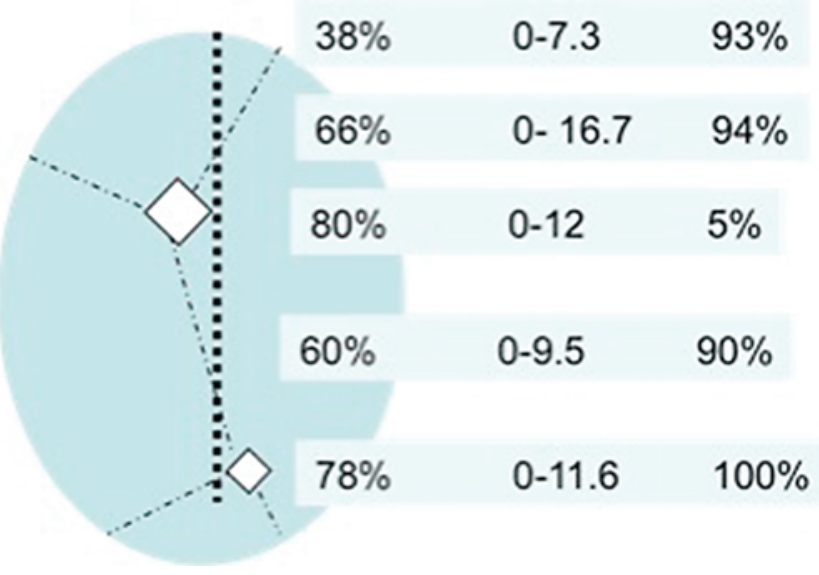

FIG. 5. Scheme representing the frequency of sinus displacement at the 5 landmark points (first column), the range of its deviation (second column), and the percentage of cases where the sinus is contralateral to the synostosis side (third column). SSS bregma (anterior) and lambda (posterior). Figure is available in color online only.

between these features was not observed. Only 1 patient presented with a smaller ventricle on the contralateral side of the synostosis (Fig. 7).

\section{Discussion}

Sagittal suture is usually used as an external landmark for the location of the midline portion of the SSS. ${ }^{6,17} \mathrm{In}$ tracranial venous structures have received increasing attention from neurosurgeons, especially when detachment of part of the SSS during surgery is needed. Moreover, even in minimally invasive endoscopic techniques, there is a close relationship with the SSS because incisions are performed anteriorly from the anterior fontanelle to the pterion on the side of the fused suture and posteriorly in contact with lambda. ${ }^{6,17}$ Thus, in both open and endoscopic approaches the surgeons need to be careful with the SSS to ensure the safest possible surgery.

Tubbs et al. ${ }^{19}$ have shown that in the majority of specimens, the SSS tends to deviate to the right of the midline at a distance no more than $11 \mathrm{~mm}$. Samadian et al. ${ }^{16}$ found a maximum deviation of $10 \mathrm{~mm}$, also to the right side. These studies were performed in cadaveric specimens without skull deformities or hyperostosis. Similarly, in our control group, we noticed that among 7 patients who presented with a deviation of the SSS, in all cases it was to the right side. The largest distance was at the level of the midpoint bregma-lambda $(7.1 \mathrm{~mm})$. In our study, the sinus was located just below the suture in the majority of control

TABLE 1. Differences between the mean distances of UCS and control groups

\begin{tabular}{cccc}
\hline & $\begin{array}{c}\text { Difference in Mean Distance Between } \\
\text { Groups, } \mathrm{mm}(\mathrm{SD})\end{array}$ & $95 \% \mathrm{Cl}$ & $\mathrm{p}$ Value \\
\hline Reference Landmark & $1.392(2.038)$ & $0.820-1.964$ & $<0.0001$ \\
\hline Nasion & $3.8200(3.9399)$ & $2.7143-4.9257$ & $<0.0001$ \\
\hline Bregma & $5.6330(3.7772)$ & $4.5512-6.7148$ & $<0.0001$ \\
\hline Bregma-lambda & $2.7132(2.9905)$ & $1.7907-3.6357$ & $<0.0001$ \\
\hline Lambda & $4.6140(3.3003)$ & $3.6217-5.6063$ & $<0.0001$ \\
\hline
\end{tabular}




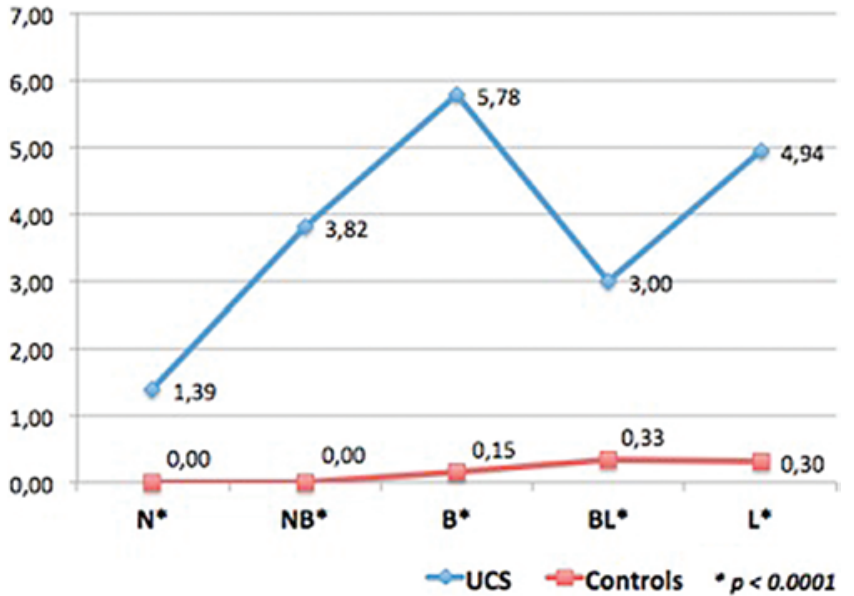

FIG. 6. Graphic representation of median distance from suture to sinus in 5 landmarks. $\mathrm{B}=$ bregma; $\mathrm{BL}=$ midpoint bregma-lambda; $\mathrm{L}=$ lambda; $\mathrm{N}=$ nasion; $\mathrm{NB}=$ midpoint nasion-bregma. Figure is available in color online only.

subjects. Because the mean age of children in our control group was 5.5 months, the suture was still open. Thus, it was large enough to contain all extensions of the SSS, so the distances in many of these patients were 0 . This could be a limitation of the study. However, the distances found in control groups of other studies ${ }^{8,16}$ were still less significant than the distances we found in the UCS group, making our results reliable.

Concerning patients with UCS, we questioned how the SSS behaves when the skull deformity is present. To our knowledge, the study by Russell et al. ${ }^{15}$ was the only one to examine the position of the sinus relative to the sagittal suture in patients with UCS. They demonstrated a maximum deviation up to $14.4 \mathrm{~mm}$.

In the present study, we encountered a maximum deviation up to $16.7 \mathrm{~mm}$ at the midpoint nasion-bregma, showing that the metopic and sagittal sutures are not as reliable. We noticed that in patients with UCS, the nasion is displaced to the ipsilateral side of the synostosis, the bregma contralaterally, and the lambda ipsilaterally. The suture will thus have a diagonal path. However, the sinus will have a path that is usually contralateral to the displacement of the suture, trying to reach the midline designed from the cranial base, but still not aligned with this imaginary midline. Therefore, not only is the suture displaced in patients with UCS, but the SSS is also dislocated.

This corroborates the findings shown by Kehrli in 1999.7 In this thesis, the SSS goes through a plexus stage during embryonic and fetal life, derived from the notochord. Then, the SSS will develop and will be oriented cortically and dorsally by the midline signal, which is thought to be responsible for migration of the neural tube to produce cortex in the prosencephalic, mesencephalic, and rhombencephalic vesicles. On the other hand, Hemmati-Brivanlou ${ }^{5}$ showed the importance of the ectoderm to the dorsal part of the neural tube. In addition, this study showed that the fate of the dorsally located neural tube is independent of the signals that come from the axial mesoderm (notochord). In fact, a certain number of factors, similar to those belonging to the Wnt and transforming

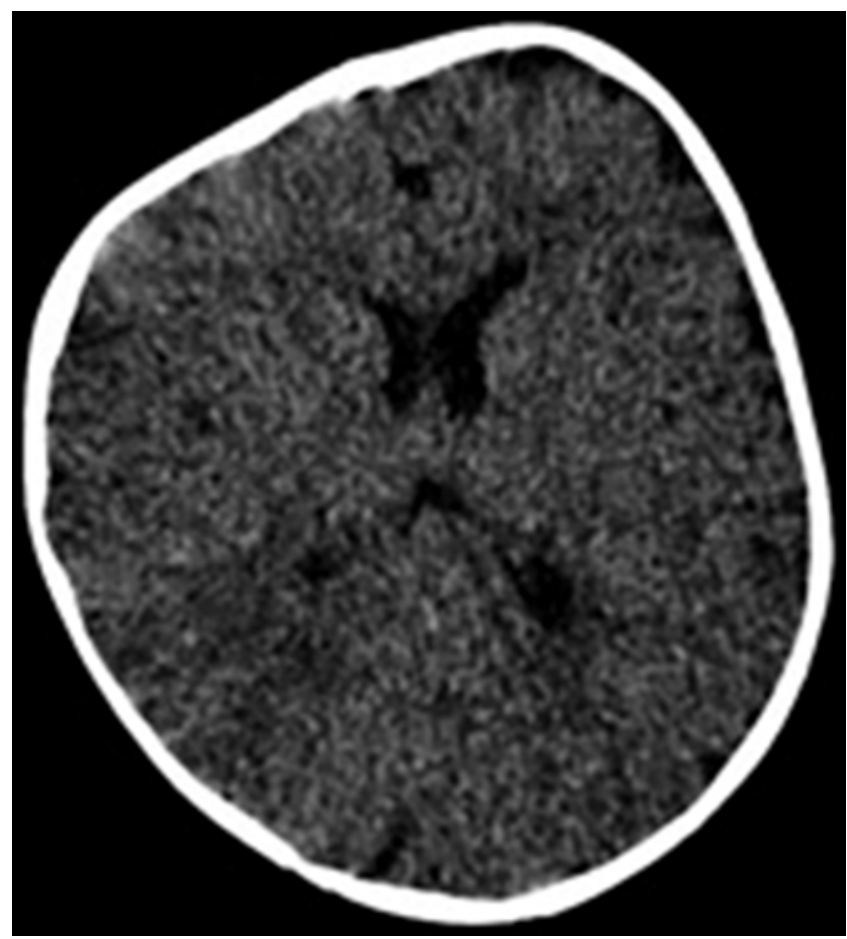

FIG. 7. Demonstration of ventricular asymmetry ipsilateral to the right UCS.

growth factor $\beta$ families, will interact to form the dorsal neural tube.

Therefore, it can be concluded from this observation that the bone signal of the midline does not seem to determine the position of the SSS, but rather the falx cerebri in between the midline of the hemispheric brain. This is confirmed by the study on development of the SSS in craniopagus twins ${ }^{8}$ and is also supported by the absence of the SSS in alobar holoprosencephaly. ${ }^{12}$ This could explain why the SSS has a different path than the suture. However, in our study, the SSS is deviated not only from the sagittal suture but also from the midline on the cranial base, as shown in Fig. 4. This finding supports the hypothesis that there is a relationship between the development of the dorsal and ventral neural tubes, which has yet to be determined by the study of the molecular basis of tissue interactions. Considering these factors and as confirmed by our data in the control group, the sagittal suture may be a reliable landmark when there is an equilibrium between dorsal and ventral neural tube development.

In this study, all patients referred to our department had a CT scan before treatment. Therefore, the study represents the full spectrum of our population.

In relation to the extracerebral space, we found that $38 \%$ of patients presented with an extracerebral fluid collection, which was always located contralaterally to the synostosis. This collection was small in all cases and not related to deviation of the sinus. Ventricular asymmetry was found in $52 \%$ of patients; in $96 \%$ of these cases, the asymmetry was located ipsilaterally to the synostosis. Also, no correlation was found between sinus displacement and ventricular asymmetry. We did not find any studies in the literature reporting the same finding, suggesting that it needs 
to be studied more carefully. Moreover, we did not notice a relationship between the degree of SSS displacement and the type of UCS (Di Rocco classification ${ }^{3}$ ).

\section{Conclusions}

Craniosynostosis results from abnormalities in the equilibrium of the cranial suture complex, which is composed of the dura mater underlying the suture, the osteogenic fronts of the calvarial bone plates, the intervening cranial suture mesenchyma, and the overlying pericranium. Although significant progress has been made in our understanding of the development of this pathology, tissue interaction between the cranial suture and the SSS has yet to be determined. For both open and endoscopic approaches, neurosurgeons should be aware that the sagittal suture is not reliable, as expected, as a landmark for the SSS in patients with UCS. We recommend that neurosurgeons carefully analyze preoperative imaging and consider that the SSS might be as far as $17 \mathrm{~mm}$ from the suture in patients with UCS. Thus, we suggest that the safety margin during surgery should be $20 \mathrm{~mm}$ from this point, using a secure distance of $37 \mathrm{~mm}$ from the sagittal suture.

\section{References}

1. Di Rocco F, Arnaud E, Meyer P, Sainte-Rose C, Renier D: Focus session on the changing "epidemiology" of craniosynostosis (comparing two quinquennia: 1985-1989 and 2003-2007) and its impact on the daily clinical practice: a review from Necker Enfants Malades. Childs Nerv Syst 25:807-811, 2009

2. Di Rocco F, Arnaud E, Renier D: Evolution in the frequency of nonsyndromic craniosynostosis. J Neurosurg Pediatr 4:21-25, 2009

3. Di Rocco C, Paternoster G, Caldarelli M, Massimi L, Tamburrini G: Anterior plagiocephaly: epidemiology, clinical findings, diagnosis, and classification. A review. Childs Nerv Syst 28:1413-1422, 2012

4. Foltz EL, Loeser JD: Craniosynostosis. J Neurosurg 43:4857, 1975

5. Hemmati-Brivanlou A: La morphogènése du systèmenerveux chez les vertébrés. Med Sci 16:150-158, 2000

6. Jimenez DF, Barone CM: Endoscopic techniques for craniosynostosis. Atlas Oral Maxillofac Surg Clin North Am 18:93-107, 2010

7. Kehrli P: Le Sinus Sagittal Supérieur. Etude Embryologique, Histologiqueetanatomique, vol 1 [doctoral thesis]. Strasbourg, France: Université Louis Pasteur, 1999

8. Lasjaunias P, Kwok R, Goh P, Yeong KY, Lim W, Chng SM: A developmental theory of the superior sagittal sinus(es) in craniopagus twins. Childs Nerv Syst 20:526-537, 2004

9. Lattanzi W, Bukvic N, Barba M, Tamburrini G, Bernardini C, Michetti F, et al: Genetic basis of single-suture synostoses: genes, chromosomes and clinical implications. Childs Nerv Syst 28:1301-1310, 2012

10. Mathijssen I, Arnaud E, Lajeunie E, Marchac D, Renier D: Postoperative cognitive outcome for synostotic frontal plagiocephaly. J Neurosurg 105 (1 Suppl):16-20, 2006

11. Matushita H, Alonso N, Cardeal DD, de Andrade F: Frontalorbital advancement for the management of anterior plagiocephaly. Childs Nerv Syst 28:1423-1427, 2012

12. Probst FP: [Basic morphology and diagnosis of the prosencephaly malformation (author's transl).] Radiologe 21:497506, 1981 (Ger)

13. Renier D, Lajeunie E, Arnaud E, Marchac D: Management of craniosynostoses. Childs Nerv Syst 16:645-658, 2000

14. Rhoton AL Jr: The cerebral veins. Neurosurgery 51 (4 Suppl):S159-S205, 2002

15. Russell AJ, Patel KB, Skolnick G, Woo AS, Smyth MD: The path of the superior sagittal sinus in unicoronal synostosis. Childs Nerv Syst 30:1701-1709, 2014

16. Samadian M, Nazparvar B, Haddadian K, Rezaei O, Khormaee F: The anatomical relation between the superior sagittal sinus and the sagittal suture with surgical considerations. Clin Neurol Neurosurg 113:89-91, 2011

17. Sayhan S, Guvencer M, Ozer E, Arda MN: Morphometric evaluation of parasagittal venous anatomy for intracranial approaches: a cadaveric study. Turk Neurosurg 22:540-546, 2012

18. Slater BJ, Lenton KA, Kwan MD, Gupta DM, Wan DC, Longaker MT: Cranial sutures: a brief review. Plast Reconstr Surg 121:170e-178e, 2008

19. Tubbs RS, Salter G, Elton S, Grabb PA, Oakes WJ: Sagittal suture as an external landmark for the superior sagittal sinus. J Neurosurg 94:985-987, 2001

\section{Disclosures}

The authors report no conflict of interest concerning the materials or methods used in this study or the findings specified in this paper.

\section{Author Contributions}

Conception and design: all authors. Acquisition of data: Protzenko Cervante, Arnaud, Brunelle. Analysis and interpretation of data: all authors. Drafting the article: all authors. Critically revising the article: all authors. Reviewed submitted version of manuscript: all authors. Approved the final version of the manuscript on behalf of all authors: Protzenko Cervante. Statistical analysis: Protzenko Cervante, Arnaud, Di Rocco. Administrative/ technical/material support: Arnaud, Brunelle, Di Rocco. Study supervision: Arnaud, Brunelle, Di Rocco.

\section{Correspondence}

Tatiana Protzenko Cervante, Department of Pediatric Neurosurgery, Hôpital Necker Enfants Malades, 149 Rue de Sèvres, Paris 75015, France. email: tatiana.protzenko@gmail. com. 